\title{
Measurements of scalar released from point sources in a turbulent boundary layer
}

K. M. Talluru ${ }^{1}$, C. Hernandez-Silva ${ }^{1}$. J. Philip ${ }^{2}$ and K. A. Chauhan $^{1}$

${ }^{1}$ School of Civil Engineering

The University of Sydney, NSW 2006, AUSTRALIA

${ }^{2}$ Department of Mechanical Engineering,

The University of Melbourne, VIC 3010 AUSTRALIA

E-mail: murali.talluru@sydney.edu.au 


\begin{abstract}
Measurements of velocity and concentration fluctuations for a horizontal plume released at several wall-normal locations in a turbulent boundary layer (TBL) are discussed in this paper. The primary objective of this study is to establish a systematic procedure to acquire accurate single-point concentration measurements for a substantially long time so as to obtain converged statistics of long tails of probability density functions of concentration. Details of the calibration procedure implemented for long measurements are presented, which include sensor drift compensation to eliminate the increase in average background concentration with time. While most previous studies reported measurements where the source height is limited to, $s_{z} / \delta \leq 0.2$, where $s_{z}$ is the wall-normal source height and $\delta$ is the boundary layer thickness, here results of concentration fluctuations when the plume is released in the outer layer are emphasized. Results of mean and root-mean-square (r.m.s.) profiles of concentration for elevated sources agree with the well-accepted reflected Gaussian model [Fackrell and Robins, 1982, J. Fluid. Mech., vol. 117]. However, there is clear deviation from the reflected Gaussian model for source in the intermittent region of TBL particularly at locations higher than the source itself. Further, we find that the plume halfwidths are different for the mean and r.m.s. concentration profiles. Long sampling times enabled us to calculate converged probability density functions at high concentrations and these are found to exhibit exponential distribution.
\end{abstract}

\title{
1. Introduction
}

Environmental concerns due to the spread of urban areas increasingly demand better prediction of hazardous gases released from a source above the ground in the atmospheric boundary layer. The risks associated with the dispersion of atmospheric contaminants can be broadly classified into two categories - chronic and accidental. Chronic risks may be estimated using the information of mean concentration field while in the case of accidental risks, one has to properly evaluate potential health hazards from incidental exposure to excessive concentration levels. Due to the highly turbulent and intermittent nature of the mixing process, regions of high concentration exist within the plume and often significantly exceed the mean value. It is such extreme events that can cause extensive damage to environment, for example, spontaneous ignition of a gas can occur and may lead to accidental fires, when the instantaneous concentration level of a gas exceeds its flammability limit. For this, detailed information of scalar variance and probability distribution functions (PDFs) of scalar fluctuations is required.

Although prior knowledge of scalar statistics such as PDFs are most desirable, they are difficult to obtain in practice, as steady flow conditions and long sampling times are required to ensure stationarity and convergence. In this regard, measuring concentration fluctuations in wind tunnels and water channels is preferred over outdoor measurements as stationary turbulence can be maintained over sufficiently long periods to enable reliable measurements of the statistical quantities. However, [Yee et al., 1993] pointed out that the large-scale eddy motions that are responsible for plume meandering are not properly represented in laboratory wind tunnels, due to their small sizes. Hence, large wind tunnels are a prerequisite to generate turbulent boundary layers (TBL) at sufficiently high Reynolds number, where large-scale motions are observed [Hutchins and Marusic, 2007b]. Consequently, there have been experimental studies of concentration using a point source tracer gas in an open-circuit [Fackrell and Robins, 1982] and closed-loop [Nironi et al., 2015] laboratory boundary layer wind tunnels. A common problem experienced in concentration measurements in 
wind tunnels is that the background concentration continuously changed with time, especially in closed-loop tunnels, which affected the accuracy of measurements, in particular, the PDFs. It is not clear how this problem has been dealt in the previous measurements.

In practice, two experimental techniques are widely used for continuous measurement of concentration, namely, flame ionisation detection (FID) and photoionisation detection (PID). Both techniques have similar operating principle of ionising a hydrocarbon gas and measuring the voltage output, which in turn is a function of the concentration of ions present in the flame/ionisation chamber. In FID, one uses flame to ionise the gas while in PID high intensity ultra violet light is utilized. The problem of drift in sensor output, either due to internal electronics or due to change in background concentration is of concern in both these measurement techniques. Typically, sensors are calibrated twice each day depending on the consistency of flame temperature in FID and it is assumed that the background concentration changes linearly with time, which is later corrected during post-processing [Nironi et al., 2015]. There is a need for systematic calibration procedures for both PID and FID sensors and such a procedure for PID is elaborately discussed in the present study.

Fackrell and Robins [1982] and Nironi et al. [2015], both using FID technique, have shown that the mean and variance of concentration fluctuations in an artificially stimulated (using roughness elements) turbulent boundary layer exhibit either reflected Gaussian or Gaussian behaviour depending on the source height. Fackrell and Robins [1982] reported that a ground level plume tends to disperse in a Gaussian distribution (to a close approximation) while an elevated plume exhibits reflected Gaussian behaviour. However, both studies considered a single ground level source and a single elevated source and hence could not study if there is any influence of the source location on the plume spread. Further, it is noted that in these studies, the interaction between the plume and the so-called 'canonical' turbulent boundary layer is rarely investigated. Recent developments in the structure of TBL have provided significant insights on the role of large-scale structures in (i) transporting momentum and (ii) modulating fine-scale turbulence close to the surface [Smits et al., 2011a, Hutchins and Marusic, 2007a,b, Marusic et al., 2010, and references therein]. As stated earlier, it is these large-scale motions that are responsible for plume meandering and hence there is a need for experimental studies to understand the interaction between the structure of TBL and a passive scalar plume. As a first step towards understanding this interaction, we implement procedures to simultaneously measure velocity and concentration. Although it is known that smooth, flat plate boundary layer has only a slight similarity to an atmospheric boundary layer, we conducted these wind tunnel measurements, first, to understand if there is any effect of outer region intermittency on scalar behaviour. Secondly, to test the performance of the present instrumentation in the region of turbulent boundary layer, where convection velocity is quite high since we aim to extend the study to different terrain categories (e.g. urban, suburban, grasslands, etc), where measurements at such heights would be still within the fully turbulent region.

\section{Experimental details}

The experiments are performed in the closed-loop Boundary Layer Wind Tunnel (BLWT) in the School of Civil Engineering at the University of Sydney. The working section of the wind tunnel is $19 \mathrm{~m}$ long and is $2.5 \mathrm{~m}$ wide. It has a constant roof height 


$\begin{array}{cl}\text { Symbol } & \text { Definition } \\ x & \text { Streamwise distance } \\ z & \text { Wall-normal distance } \\ U_{\infty} & \text { Freestream velocity } \\ \delta & \text { Boundary layer thickness } \\ U_{\tau} & \text { Friction velocity } \\ \nu & \text { Kinematic viscosity of air } \\ R e_{\theta} & \text { Reynolds number based on momentum thickness } \\ R_{\tau} \text { or } \delta^{+} & \text {Friction Reynolds number } \\ U & \text { Time-averaged velocity } \\ u & \text { Fluctuating velocity } \\ l & \text { Length of hotwire } \\ d_{\mathrm{hw}} & \text { Diameter of hotwire } \\ d_{\mathrm{PID}} & \text { Diameter of PID inlet } \\ s_{d} & \text { Source diameter of tracer gas outlet. } \\ s_{u} & \text { Source velocity } \\ s_{z} & \text { Wall-normal height of point scalar source } \\ s_{x} & \text { Distance between the scalar source and the } \\ \rho_{\text {air }} & \text { measurement station } \\ \rho_{g a s} & \text { density of air at NTP conditions (101.325 kPa and } 293.15 \mathrm{~K}) \\ D & \text { density of gas mixture at NTP conditions } \\ S c & \text { Molecular diffusivity of tracer gas } \\ \tilde{C} & \text { Schmidt number of tracer gas }=\nu / D \\ C & \text { Instantaneous concentration } \\ c & \text { Time-averaged concentration } \\ \sqrt{\overline{c^{2}}} & \text { Fluctuating concentration, mean subtracted } \\ & \text { r.m.s of concentration fluctuations } \\ & \end{array}$

Table 1. Nomenclature

of $2.0 \mathrm{~m}$ in the region, $0 \leq x \leq 12 \mathrm{~m}$, followed by an arrangement of horizontal slats in the roof which can be adjusted to control the streamwise pressure gradient between $x=12 \mathrm{~m}$ and $x=17 \mathrm{~m}$. Here $x$ is the distance from the trip, a $50 \mathrm{~mm}$ wide P60 grit sandpaper spanning the full width of the tunnel to trigger transition to turbulence of the incoming laminar boundary layer. The flow is driven by a $250 \mathrm{~kW}$ fan. Flow induced by the fan passes through a settling chamber comprised of a flow-straightener and a series of mesh screens before entering the contraction (with an area ratio of 4.5:1) and then the test section.

The free-stream velocity $\left(U_{\infty}\right)$ can be varied between 0 and $27 \mathrm{~m} / \mathrm{s}$. With the wind tunnel in the configuration for present study, the free-stream turbulence intensity is approximately $0.5 \%$ for a free-stream velocity range of $0-27 \mathrm{~m} / \mathrm{s}$. The long development section permits growth of very thick boundary layers. For the current study, the free stream velocity is nominally set to $10 \mathrm{~m} / \mathrm{s}$, which resulted in a 


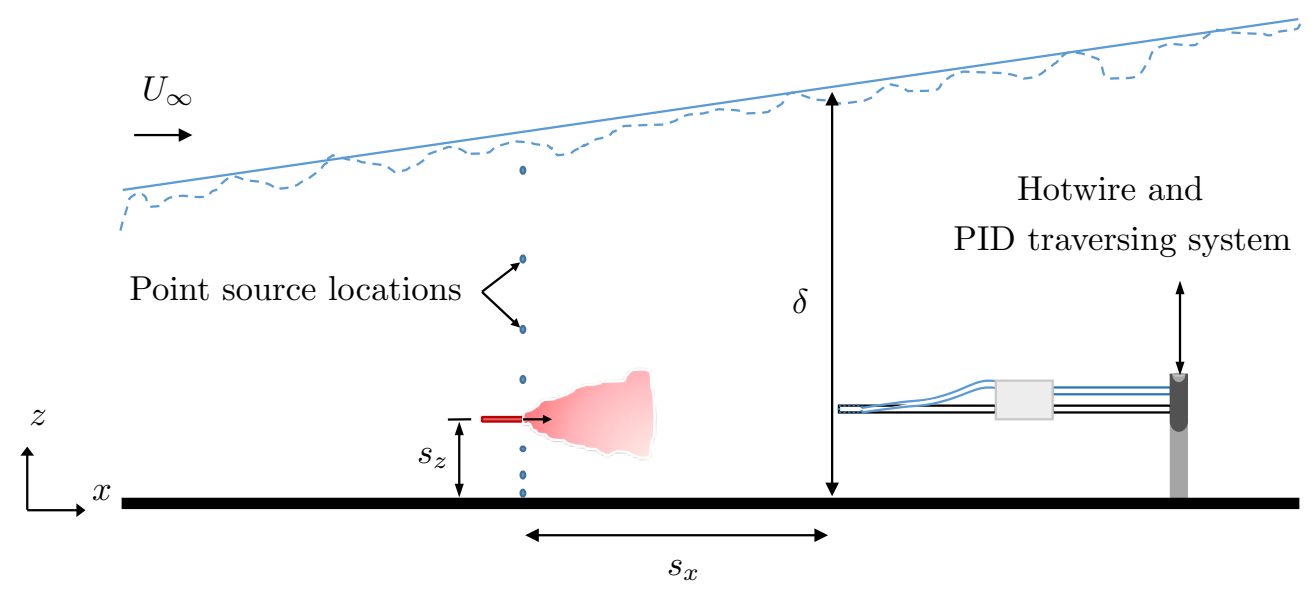

(a) Side view

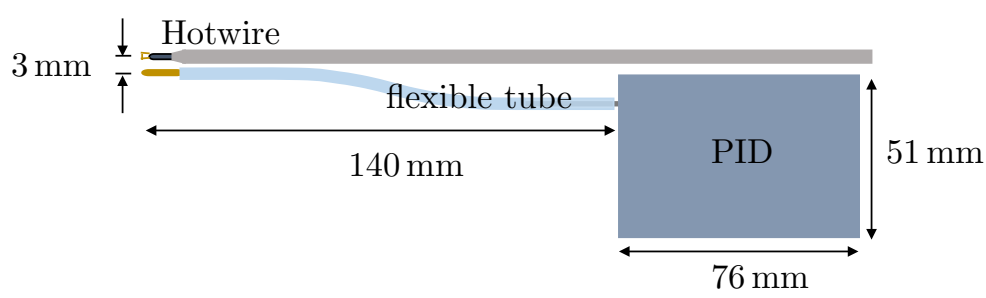

(b) Top view

Figure 1. Schematic of experimental setup: (a) Side view and (b) Top view showing the details of hotwire and PID arrangement.

boundary layer thickness, $\delta=0.31 \mathrm{~m}$ (determined from a fit to the composite profile of Chauhan et al. [2009]) and friction velocity, $U_{\tau}=0.37 \mathrm{~m} / \mathrm{s}$ (determined by matching the logarithmic mean velocity profile to the constants $\kappa=0.384$ and $A=4.17$ ) at the measurement location, $x=13 \mathrm{~m}$ downstream of the trip. This yields a friction Reynolds number, $R e_{\tau}=\delta^{+}=\delta U_{\tau} / \nu \approx 7850$ ( $\nu$ is the kinematic viscosity of air) at the measurement location. Throughout this paper, $x$ and $z$ refer to the streamwise and wall-normal directions while $u$ denotes the streamwise fluctuating velocity component. All the other symbols used in this paper are defined in table 1.

A mild favourable pressure gradient, equivalent to an increase of $3 \%$ in the freestream velocity over a streamwise distance of $12 \mathrm{~m}$, is observed in the tunnel due to a constant rectangular cross-section over this distance. This pressure gradient corresponds to Clauser pressure gradient parameter $\beta=-0.0524$ and thus is a very weak pressure gradient. Here, $\beta$ is defined as $\frac{\delta^{*}}{\tau_{w}} \frac{d P}{d x}$, where $\delta^{*}$ is the displacement thickness, $\tau_{w}$ is the wall shear stress and $\frac{d P}{d x}$ is the streamwise pressure gradient. Other parameters of the boundary layer are listed in table 2. Note that normalisation using wall units is denoted by a ' + ' symbol in this paper, for example, $l^{+}=l /\left(\frac{\nu}{U_{\tau}}\right)$ and $U^{+}=U / U_{\tau}$.

Simultaneous measurements of velocity and concentration are made using a single hotwire and a photo-ionisation detector. A schematic of the experimental setup is 


\begin{tabular}{|lc|l|l|}
\hline $\begin{array}{l}\text { source height } \\
s_{z} / \delta\end{array}$ & Symbols & $\begin{array}{l}\text { Boundary layer } \\
\text { properties at } x=13 \mathrm{~m}\end{array}$ & $\begin{array}{l}\text { Tracer gas mixture } \\
\left(1.5 \% \mathrm{C}_{4} \mathrm{H}_{8}+98.5 \% \mathrm{~N}_{2}\right)\end{array}$ \\
\hline $32 / \delta^{+}$ & $\bigcirc$ & $l^{+} \approx 24$ & \\
$350 / \delta^{+}$ & $\diamond$ & $d_{\mathrm{PID}}^{+} \approx 18$ & \\
0.1 & $\triangle$ & $\delta^{+}=R e_{\tau} \approx 7850$ & $\rho_{\text {air }}=1.205 \mathrm{~kg} / \mathrm{m}^{3}$ \\
0.25 & $\nabla$ & $\operatorname{Re}_{\theta}=14300$ & $\rho_{\text {gas }}=1.185 \mathrm{~kg} / \mathrm{m}^{3}$ \\
0.33 & $\triangleleft$ & $U_{\infty}=10.2 \mathrm{~m} / \mathrm{s}$ & $D=9.9 \times 10^{-6} \mathrm{~m}^{2} / \mathrm{s}$ \\
0.5 & $\triangleright$ & $U_{\tau}=0.367 \mathrm{~m} / \mathrm{s}$ & $S c=1.52$ \\
0.67 & $\square$ & $s_{d}^{+} \approx 38$ & \\
$\delta_{99} / \delta$ & $\downarrow$ & $\delta=0.310 \mathrm{~m}$ & \\
\hline
\end{tabular}

Table 2. Experimental parameters. See table 1 for nomenclature. $(+)$ represents normalisation using viscous length scale, for instance, $l^{+}=l U_{\tau} / \nu$. Here $R_{\theta}=$ $\frac{\theta U_{\infty}}{\nu}$, where $\theta$ is the momentum thickness defined as $\int_{o}^{1} \frac{U\left(U_{\infty}-U\right)}{U_{\infty}^{2}} d\left(\frac{z}{\delta}\right)$.

shown in figure 1 , where various parameters are also shown. The hotwire and PID sensors are mounted on a vertical traversing system at a spanwise separation of $3 \mathrm{~mm} \approx$ $4 d_{\text {PID }}$, where $d_{\text {PID }}=0.76 \mathrm{~mm}$ is the inner diameter of PID inlet tube. This spacing is in accordance with figure 5 of Metzger and Klewicki [2003], who showed that suction of PID has negligible influence on the adjacent hotwire if they are separated by a distance greater than $3 d_{\mathrm{PID}}$. More details of hotwire and PID instrumentation are discussed in $\S 3$.

A point source tracer gas ( a mixture of $1.5 \%$ iso-butylene and $98.5 \%$ Nitrogen) is released at eight different heights $\left(s_{z}\right.$, see table 2) above the floor at a streamwise separation, $s_{x} / \delta=1$, upstream of the measurement location. The flow rate of the tracer gas is adjusted to match the exit velocity with the local boundary layer mean velocity at $z=s_{z}$. It should be noted that the concentration measurements here are only acquired over a limited region of the boundary layer and not across its entire thickness. For this, preliminary concentration measurements were performed with coarsely spaced points and short sampling times to get an estimate of plume-widths at different heights. Thereafter, long sampling time measurements at closely spaced 30 points within the initially estimated plume width are performed and only those results are discussed here. A sampling time of $T=300 \mathrm{sec}$ is used in the current study, which is normalised using $U_{\infty}$ and $\delta$ to give boundary layer turn over times, i.e., $T U_{\infty} / \delta \approx$ 9700 , in our measurements. For converged statistics, these numbers need to be large. The largest structures in boundary layers can commonly exceed $20 \delta$ [Hutchins et al., 2009], and we would typically require several hundreds of these events to advect past the sensor array before we could expect converged statistics. The chosen sampling time is found to yield converged statistics of velocity spectra as well as the long tails of PDFs of concentration. 


\section{Instrumentation}

\subsection{Hotwire anemometry}

Hotwire anemometry is used to measure velocity fluctuations in these experiments. The single-wire probe is a Dantec 55P15 type sensor and has a spacing of $1.05 \mathrm{~mm}$ with 5 micron diameter $\left(d_{\mathrm{hw}}\right)$ Platinum coated Tungsten wire welded between the prongs. The length $(l)$ to diameter ratio of the hotwire is around 210, keeping with the recommendations of Ligrani and Bradshaw [1987] and Hutchins et al. [2009]. The inner-normalised sensor length $\left(l^{+}\right)$of hotwire is approximately 24 and is understood to have comparable spatial resolution as that of a previous study by Marusic et al. [2015]. In order to correct the spatial averaging effects in streamwise turbulence intensity, one can refer to the correction scheme proposed by Smits et al. [2011b]. The single-wire is operated using a Dantec constant temperature anemometer at an overheat ratio of 1.8. For most measurements, the filter cut-off frequency is set at $10 \mathrm{kHz}$ and the data is sampled at $20 \mathrm{kHz} /$ channel and is digitised using a 24-bit National Instruments A/D card, NI 4303.

Calibration of the hotwire is performed in situ to eliminate the need for the probe to be moved between calibration and boundary-layer measurement. Hotwire is calibrated before and after each experiment against a Dantec reference velocity probe located in the undisturbed free stream flow. We used a reference velocity probe instead of a Pitot-static tube as a hot-wire calibration device for the following reasons: First, we noted that Pitot-static tube measurements were less reliable at low speeds due to the limitations of pressure transducer, while the reference probe, once calibrated provided reliable and consistent readings. Second, the accuracy of Pitot-static tube measurements depended on the accuracy of pressure and temperature transducers that are used to deduce velocity information, while a reference velocity probe is temperature compensated and provided accurate measurements. Lastly, a reference velocity probe has better frequency response compared to that of a Pitot-static tube, whose response is primarily limited due to the tubing between Pitot-static tube and pressure transducer. A third-order polynomial is fitted for the functional relation between measured hotwire voltage and corresponding velocity during calibration. Besides velocity, temperature is continuously measured in the free stream for the entire duration of an experiment using a fast response copper tip RTD (resistive temperature device) sensor by Omega, Inc. with a response time of 1 sec. It is noticed that the temperature of the flow gradually increased by approximately $1.5^{\circ} \mathrm{C}$ over a three hour long experiment. Hence, a temperature correction scheme (as outlined in Talluru et al. [2014]) is employed to account for drift in the hotwire voltage over the course of an experiment.

The systematic error in velocity measurements using hotwire is primarily affected by its calibration, and so, it is estimated based on the accuracy of Dantec reference velocity probe (with a resolution of $0.05 \mathrm{~m} / \mathrm{s}$ ) and temperature transducer (with a resolution of $0.01^{\circ} \mathrm{C}$ ). On the other hand, random error (which is primarily due to sensor drift) in the measurements is measured as the percentage of repeatability of a given experiment under identical conditions. Overall, it is found that the systematic error is about $\pm 2 \%$, while the random error is close to $\pm 1 \%$ in velocity measurements. 


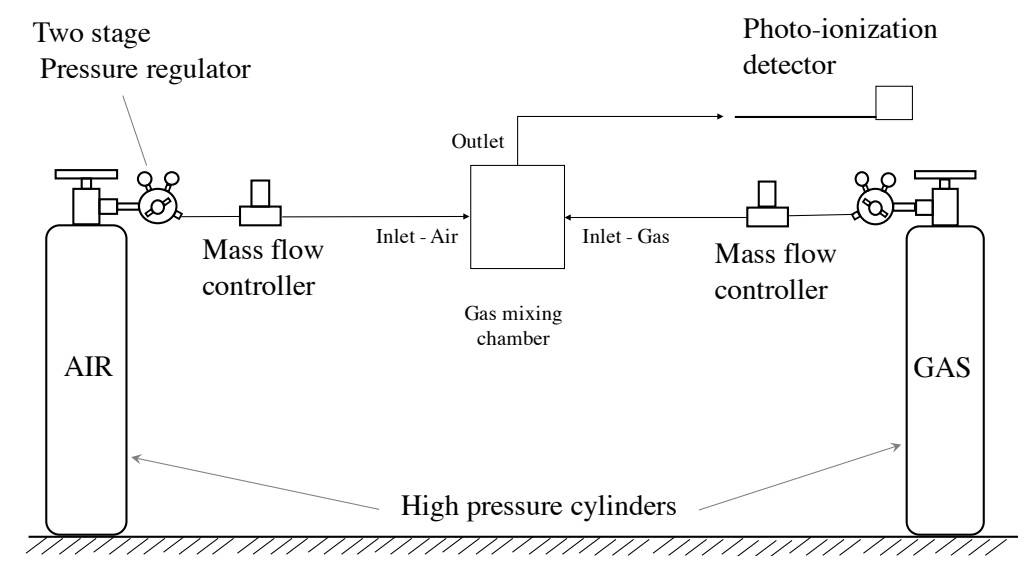

Figure 2. A schematic of the PID calibration system.

\subsection{Photo-ionisation detector (PID)}

Measurements of instantaneous concentration are obtained using a fast-response photo-ionisation detector manufactured commercially by Aurora Scientific, Inc. The PID, as shown in figure 1, consists of inlet tube, sensor head, ultra-violet (UV) lamp, electronics, a pump and a pre-amplifier. The technique of PID and its use in measuring concentration fluctuations dates back to the studies by Chandler [1991] and Yee et al. [1993], and since then has undergone several changes leading to the present one. Metzger and Klewicki [2003] combined the techniques of PID and constant temperature hotwire anemometry to develop a scalar transport probe to simultaneously measure gradients of concentration and two components of velocity, respectively. They extensively documented flow disturbance due to sensor head, frequency response and spatial resolution of the sensor and hence these details will not be discussed here. In order to further alleviate the flow disturbances due to sensor head, the inlet needle of PID is modified in the current study; the inlet needle is placed further away from the sensor head using a short length of flexible tubing, as shown in figure 1. However, adding a flexible tube has led to slight reduction in the frequency response of PID. Hence, the final tube length is chosen as a trade-off between blockage effects and sensitivity of PID.

The operating principle of PID is outlined here. A sample of air/gas mixture is drawn into the detection cell through an inlet tube (with diameter, $d_{\text {PID }}=0.76 \mathrm{~mm}$ and in viscous scaling, $d_{\mathrm{PID}}^{+}=18$ ) using a rotary vane pump mounted inside the controller box. A high energy UV lamp ionises gases that enter the detection cell. The detection cell and pre-amplifier are built into the sensor head and convert the gas concentration to a voltage signal. It is important to note that PID is sensitive to any gas with an ionization potential below $10.6 \mathrm{eV}$ and hence, the output of the device should be viewed as the concentration of total ionizables present. Due to this characteristic, the accuracy of PID is also dependent on the presence of any interference gases. The detection limit of PID is around $100 \mathrm{ppb}$ (parts per billion) of any other ionisable gas (iso-butylene in our study) in air and the full-scale measurement range is $500 \mathrm{ppm}$ (parts per million).

Further, the PID is designed and constructed so as to minimise flow disturbance. 
The length of the inlet tube is such that the sampling point is away from potential flow disturbances caused by the sensor head. The sensor head measures $25.4 \mathrm{~mm} \times 51 \mathrm{~mm}$ $\times 76 \mathrm{~mm}$. The diameter of the inlet tube is chosen to maximize frequency response and spatial resolution. The relevant length scale for a scalar is the Batchelor scale, defined as, $\lambda_{B}=\eta / S c^{1 / 2}$, where $\eta$ is the Kolmogorov length scale. From its definition, it is clear that the Batchelor scale is smaller than the Kolmogorov length scale by a factor of $S c^{1 / 2}$. In our measurements, the Schmidt number $(S c)$ for tracer gas is about 1.5 and so $\lambda_{B} \sim 0.82 \eta$. Taking the recommendation of Ligrani and Bradshaw [1987] and Hutchins et al. [2009] that $l^{+}<20$ is sufficient for good hotwire measurements (spatial averaging effects $<4 \%$ ), it can be inferred that $d^{+}$in the range of 16-20 would yield concentration measurements of similar spatial resolution. Overall, both hotwire and PID used in our experiments have limitations in fully resolving velocity and concentration close to the wall but have very good spatial resolution in the outer region of TBL.

The true frequency response of the PID sensor is measured using a custom built facility that consists of a chopper wheel delivering a quasi-impulse of tracer gas to the PID sensor. It is found that the frequency response of PID is dependent on the suction flow rate; $280 \mathrm{~Hz}$ at the low setting, $330 \mathrm{~Hz}$ at a medium suction rate and increasing to $400 \mathrm{~Hz}$ at the high setting. It is clear from the literature that a flame ionisation detector (FID) has similar geometry and functional performance as that of PID. Full details of the dimensions of FID can be found in Fackrell [1980]. The sampling tube has an internal diameter of $0.25 \mathrm{~mm}$ and is typically in the range of $0.2-0.3 \mathrm{~m}$ long. In a recent study, Nironi et al. [2015], reported that FID has a frequency response of $400 \mathrm{~Hz}$.

In all the measurements reported here, PID is operated at high setting and accordingly, the time-varying voltage signal from PID is filtered at $400 \mathrm{~Hz}$ using an analog filter (Krohn-Hite, Inc. USA) before it is digitised at a sampling rate of $20 \mathrm{kHz}$. The frequency response of PID $(\sim 400 \mathrm{~Hz})$ corresponds to a wavelength of $0.05 \delta$ in the log-region of a TBL when the freestream velocity is set at $10 \mathrm{~m} / \mathrm{s}$. This implies that PID is able to detect changes in concentration at two points in space that are separated by $0.05 \delta$ or more. Since the longterm goal of this experimental setup is to study the covariances between velocity and concentration, the inability of PID to resolve the fine scales in concentration is not an immediate concern.

The PID is calibrated in situ before and after every experiment using an in-house manual calibrator. The calibration setup (as shown in figure 2) consists of a gasmixing chamber, two mass flow controllers and necessary tubes and fittings. Two mass flow controllers (MFCs), manufactured by Alicat, Inc. USA, have an operating range of 0 - 2000 SCCM (standard cubic centimetre per minute) and 0-200 SCCM with an accuracy of $\pm 0.1 \%$ full scale and have typical response time of $100 \mathrm{~ms}$. The small range MFC controls the discharge flow rate from a cylinder containing $1.5 \%$ isobutylene mix (nitrogen base), while the other MFC controls the output from ultra-pure air. The calibration setup can deliver a desirable gas concentration to PID with an accuracy of $\pm 1 \%$. An example of pre- and post-calibration curves in one of the experiments is shown in figure 3 .

Since the PID voltage output is slightly non-linear, multiple-point calibration by using 6 different gas concentrations between 0 and $500 \mathrm{ppm}$ is typically performed for each experiment. The supply pressure and temperature of the two gases are equally maintained to obtain better accuracy in volumetric mixing. Calibration curves are generated by fitting a second-order polynomial between the measured voltage and 


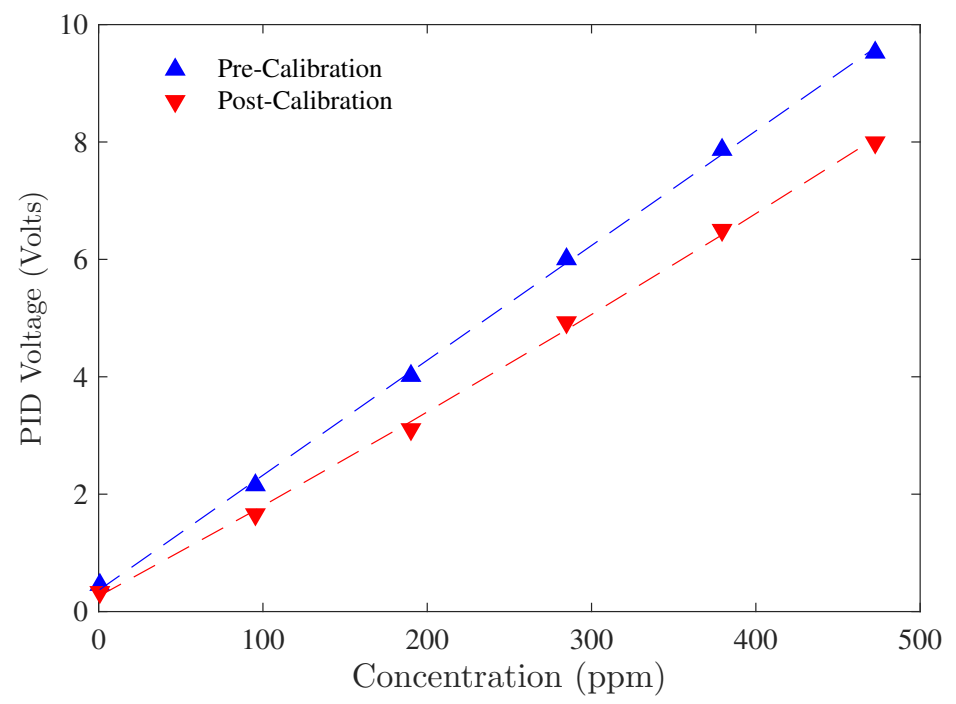

Figure 3. An example of pre- and post-calibration curves of PID. The dashed lines are second-order polynomial fits using least squares regression.

gas concentration. In our initial testing of PID, several experiments were conducted, where we continuously monitored the output voltage of PID sampling ambient air over a duration of 60 minutes each. In all these tests, we consistently observed that the output voltage of PID dropped in a linear fashion with time. This is found to be due to the gradual build up of fine dust particles on the UV lamp inside the PID. Upon cleaning the lamp, PID regained its initial voltage. Thus, it is most appropriate to conclude that there is a linear drift pattern of PID. Hence pre-calibration and post-calibration are performed and a linear interpolation in time between pre- and post-calibrations, similar to one of the methods described in Talluru et al. [2014] for correcting hotwire drift, is employed to correct drift in PID output during an experiment. Full description of the procedure for correcting drift in PID voltage is given in the Appendix.

In a similar manner, as described above for hotwire, error estimates are obtained for concentration measurements. It is found that there are higher uncertainties in concentration measurements as compared against velocity measurements. This is found to be mainly due to the drift in PID voltage during long duration experiments. In spite of correcting the drift in PID, we noted that there is repeatability error of about $\pm 2 \%$ in addition to the systematic error of $\pm 3 \%$ due to the limited resolution of PID in detecting an known concentration.

\subsection{Tracer gas and discharge tube assembly}

The tracer gas used in our study is a mixture of 1.5\% Iso-butylene and 98.5\% Nitrogen. The mixture has a relatively lower ionisation potential than most hydrocarbon gases, and this resulted in a higher voltage output from the PID for a given gas concentration. This particular gas-mixture is also chosen for the experiments since it has similar density as that of air. Gas mixture used in this study has a nominal density of 1.185 $\mathrm{kg} / \mathrm{m}^{3}$ at NTP conditions $(293.15 \mathrm{~K}$ and $101.325 \mathrm{kPa})$ while the density of air at 


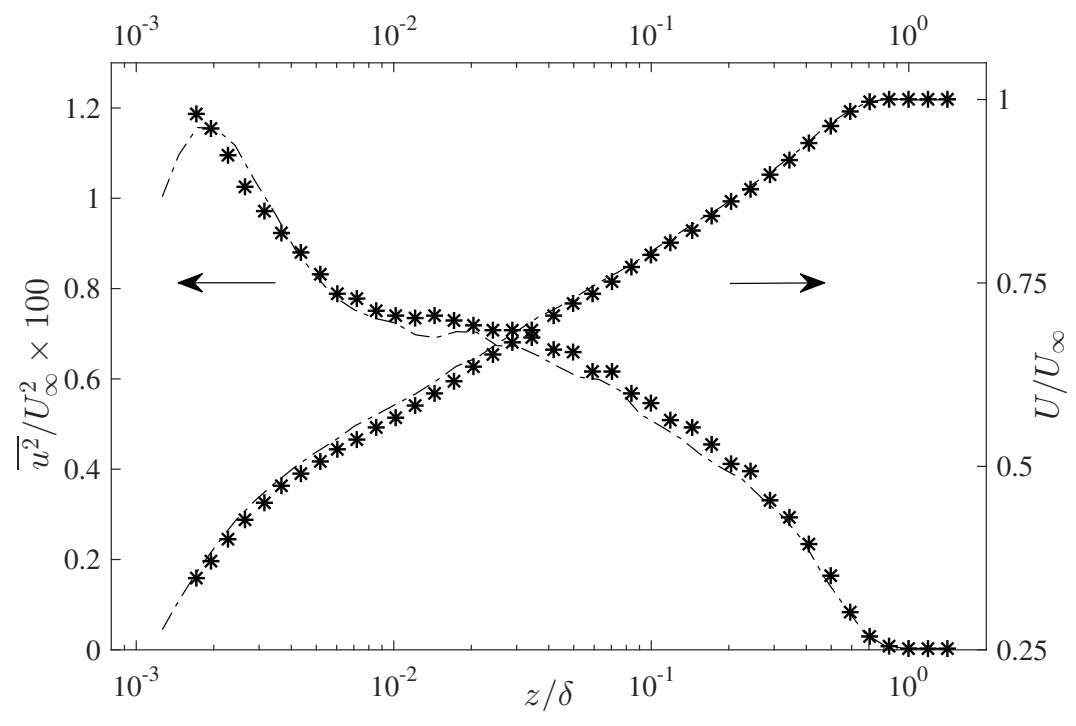

Figure 4. Comparison of mean velocity and turbulence intensity profiles using single hotwire; dot-dash lines - PID pump off and (*) - PID pump on.

similar conditions is about $1.205 \mathrm{~kg} / \mathrm{m}^{3}$. In our experiments, this corresponds to a small density difference of $1.6 \%$ between air and the gas mixture.

The tracer gas is stored in a high pressure cylinder fitted with a two-stage regulator so that the gas is discharged in the wind tunnel at nominally atmospheric pressure. The discharge tube of the tracer gas is a thin stainless steel tube with an internal diameter (ID) of $1.6 \mathrm{~mm}$. The outlet of the gas regulator is connected to the digitally controlled mass flow controller (0-2000 SCCM range). This allows to precisely match the source velocity $\left(s_{u}\right)$ of tracer gas with the mean velocity at a height $s_{z}$ in the boundary layer. In other words, the outlet velocity of the tracer gas is set as $s_{u}=U\left(z=s_{z}\right)$, often referred to as iso-kinetic condition [Nironi et al., 2015]. Throughout an experiment, the flow rate, ambient pressure and temperature in the mass flow controller are continuously monitored and recorded.

Prior to conducting scalar measurements in the turbulent boundary layer, a series of experiments are conducted to quantify the influence of PID pump operation on the mean statistics measured in the boundary layer. Two different scenarios are considered, i.e., when the PID pump is (i) off and (ii) on. A single hotwire is used to measure the mean and turbulence statistics in both cases. Results reported here have been tested for repeatability by conducting experiments on different days but maintaining the same experimental conditions. It is noticed that the measurements showed consistent values to within $\pm 1 \%$.

Looking at the mean velocity and turbulence intensity profiles shown in figure 4 , it appears that PID pump, when turned on, has a minimal influence of less than $2 \%$ on the turbulence statistics. Note that $U_{\infty}$ and $\delta$ are used for normalisation in figure 4 to avoid the error associated with measuring friction velocity, $U_{\tau}$ during PID operation. The effect seems to be evident primarily in close proximity to the wall. Similar observations were reported in the study by Metzger and Klewicki [2003].

Additional experiments are performed to quantify the influence of discharge tube (used for releasing tracer gas into the boundary layer) on the mean and turbulence 

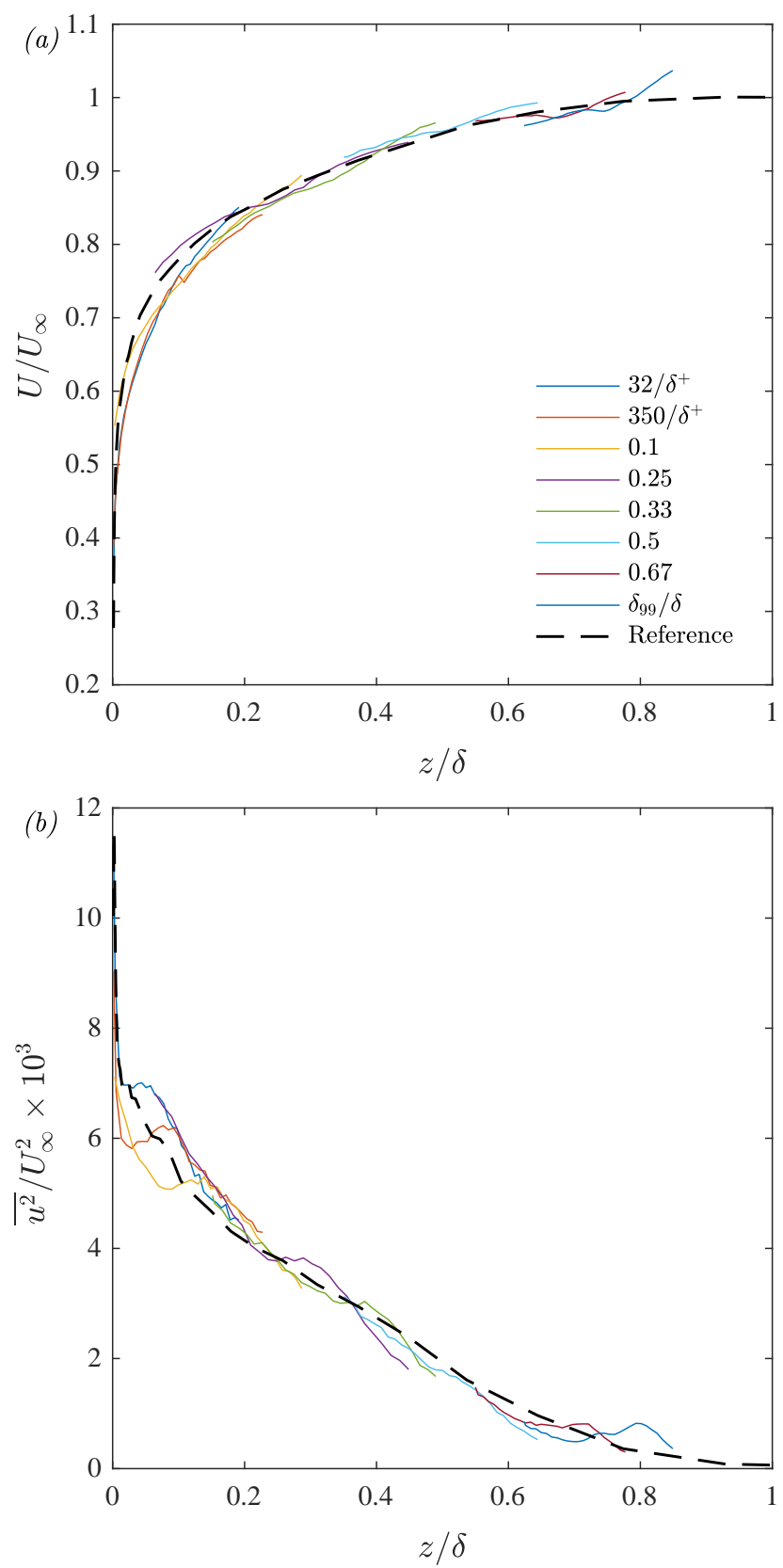

Figure 5. Comparison of (a) mean velocity and (b) turbulence intensity profiles for all source heights against the reference boundary layer measurement (black dashed lines). Colored lines represent different $s_{z}$ locations. Note that the concentration measurements here are only acquired over a limited region about the source height and not across the entire boundary layer. 


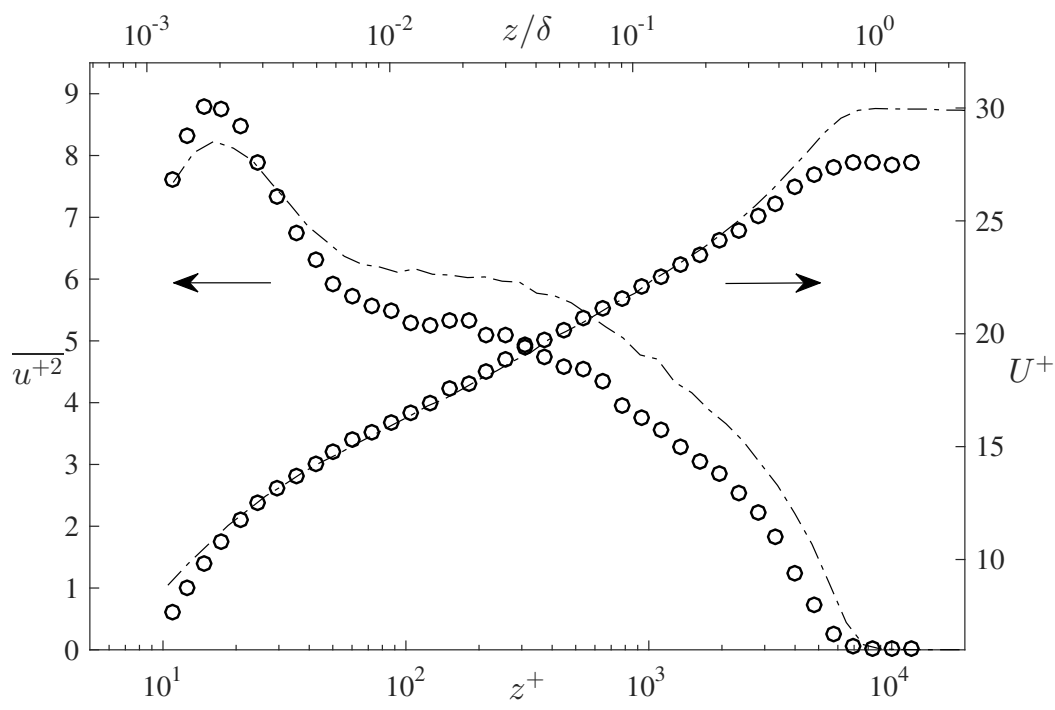

Figure 6. Comparison of mean velocity and turbulence intensity profiles in the current study $(O)$ with zero-pressure gradient boundary layer data $\left[R e_{\tau} \approx 8400\right.$; dash-dot lines Marusic et al., 2015].

statistics. Velocity measurements are taken across the TBL when the discharge tube is placed at different wall-normal locations, $z=s_{z}$, in the boundary layer. Figure 5 shows a comparison of mean velocity and turbulence intensity profiles for all configurations of $s_{z}$ against the reference case. It is evident that the discharge tube, placed $1 \delta$ upstream of the measurement location, has affected the turbulence intensity. This is primarily seen when the discharge tube is positioned at small heights above the wall, i.e, $s_{z} / \delta \leq 0.1$, where there is relatively strong mean velocity gradient in the vertical direction. There is a variation of approximately of $\pm 5 \%$ in turbulence intensity (with respect to the reference boundary layer measurement) when the source is positioned close to the wall. For all other cases, there is only minimal disturbance caused by the discharge tube.

\section{Comparison of mean and turbulence intensity profiles}

In order to establish the characteristics of the TBL in the current study, velocity measurements across the boundary layer are performed using a single hotwire. Fifty logarithmically spaced point measurements are acquired between $z=0.35$ and $550 \mathrm{~mm}$. The inner-normalised mean velocity and turbulence intensity profiles (shown in figure 6) from this experiment are compared against a similar $R e_{\tau}$ data of Marusic et al. [2015] in a zero-pressure gradient (ZPG) TBL on a smooth wall. Note that $U_{\tau}$ has been obtained by fitting the logarithmic law of the mean velocity profile with constants $\kappa=0.384$ and $A=4.17$. Dissimilarities between the two profiles are evident and these are due to a mild streamwise favourable pressure gradient (FPG) in the initial fetch of the tunnel. The results in figure 6 are consistent with observations of Harun et al. [2013], who compared a favourable, zero- and adverse pressure gradient TBLs. They reported that a FPG TBL has a smaller wake component and less energetic outer region in comparison to the ZPG case; this is also seen in our current measurements. 
Further, Harun et al. [2013] showed that the near wall peak in the turbulence intensity profile (when normalised with $U_{\tau}$ ) is smaller in a favourable pressure gradient. However, in the present study, we see higher magnitude of turbulence intensity and we suspect that this might be due to (i) the error in estimating $U_{\tau}$ and (ii) the surface of wind tunnel used in this study is not completely smooth. It is made of plywood with a roughness height (defined as $6 \sqrt{\overline{h^{\prime 2}}}$, where $h^{\prime}$ is the surface deviation about a mean roughness height) of 100 microns.

\section{Mean and Variance of concentration}

In this section we examine the distribution of mean and r.m.s. profiles of concentration for different $s_{z}$. Here we denote the instantaneous concentration, $\tilde{C}$ as the sum of its mean $(C)$ and fluctuating $(c)$ parts, i.e. $\tilde{C}=C+c$ and the concentration r.m.s as $\sqrt{\overline{c^{2}}}$. The main objective of measurements at several source heights is to examine the influence of the local structure of boundary layer on plume spread. Hence, experiments are conducted with point sources positioned in the inner, logarithmic and outer regions. Figures $7(\mathrm{a}, \& \mathrm{~b})$ show dimensional magnitude of mean and r.m.s profiles of concentration fluctuations for all values of $s_{z}$. It is clear that the spread of plume is a function of $s_{z}$. This behaviour is expected considering that mean velocity (or convection velocity) increases away from the surface while turbulent activity decreases above the buffer-layer. A tracer gas will be rapidly convected in the streamwise direction by high momentum background flow if the source is in the outer region of the boundary layer and thereby results in a narrow plume with higher mean concentration. Obviously, the dynamics of the plume spread is not this simple and will be closely linked with background turbulent motions.

Typically the plume spread is characterised by computing the vertical plume halfwidth $\left(\delta_{z}\right)$ defined as the vertical distance (with respect to $s_{z}$ ) where the maximum concentration decreases to half its value. Far from the wall, the mean and r.m.s concentration profiles can be well-approximated by a reflected Gaussian function centred at $s_{z}$. To account for the wall effect, $\delta_{z}$ is often obtained by fitting the mean and r.m.s concentration profiles to a reflected Gaussian model [Fackrell and Robins, 1982] as,

$$
C(z)=A\left[\exp \left\{-B\left(z-s_{z}\right)^{2} / \delta_{z}^{2}\right\}+\exp \left\{-B\left(z+s_{z}\right)^{2} / \delta_{z}^{2}\right\}\right],
$$

where, $B=\ln (2)$ such that $\delta_{z}$ represents the vertical half-plume width. Both simple (usual Gaussian function) and reflected Gaussian models describe the mean concentration profiles quite well for all elevated sources. Note that when $s_{z}$ is large, i.e., source is away from the wall, the second term in (1) becomes negligible and we retain the simple Gaussian function about $s_{z}$. However, the reflected Gaussian model is found to be a better fit for ground level sources. Hence, we decided to use the reflected Gaussian model for both ground level and elevated sources.

In this study $\delta_{z}$ is computed using two methods. First, by fitting the reflected Gaussian model to the mean concentration data. Second, by an alternative method in which the wall-normal locations of half of maximum concentration are identified on either side of the source height by interpolation. Two such locations (connected by vertical lines in figures $7(\mathrm{a} \& \mathrm{~b}))$ are found for all sources except for $s_{z} / \delta=32 / \delta^{+}$ and $350 / \delta^{+}$. The half-width $\delta_{z}$ is then equal to half of the distance between those two locations. For the two sources positioned close to the wall, $s_{z} / \delta=32 / \delta^{+}$and 

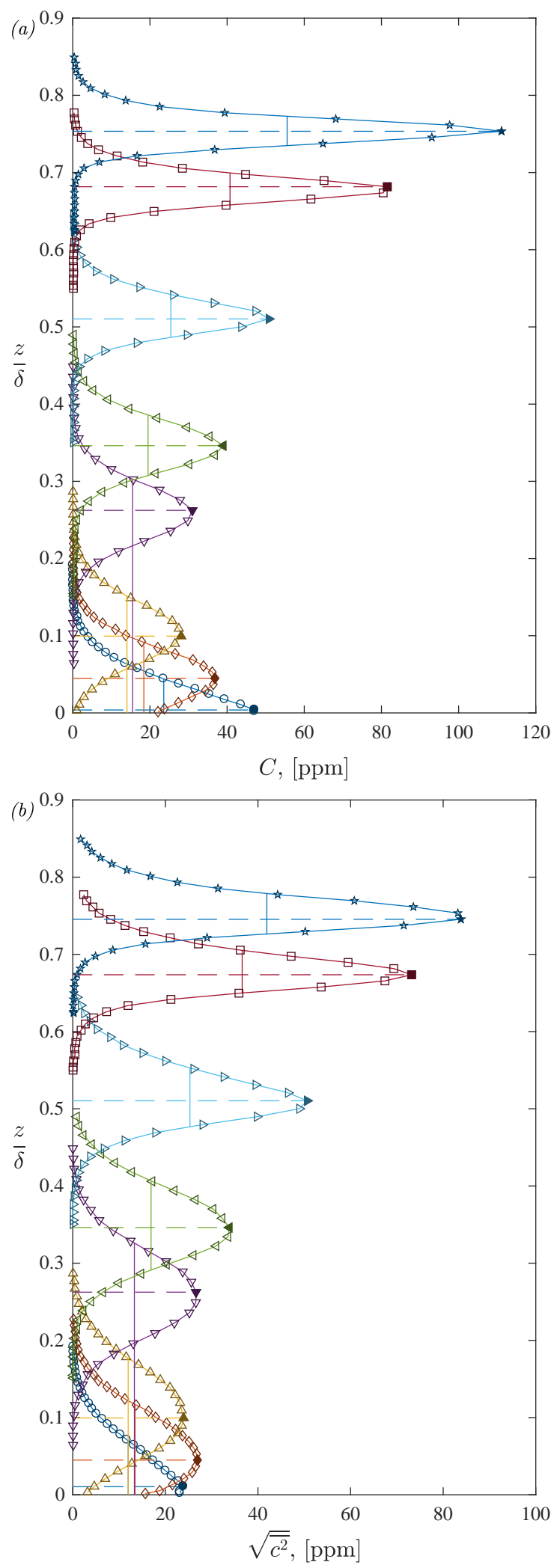

Figure 7. Profiles of (a) mean and (b) r.m.s of concentration fluctuations as a function of $z / \delta$ for all source heights. The horizontal dashed lines indicate the source heights while the vertical lines denote the locations of half the maxima in mean and r.m.s of concentration. See table 2 for symbols. Filled symbols denote the respective maxima. 


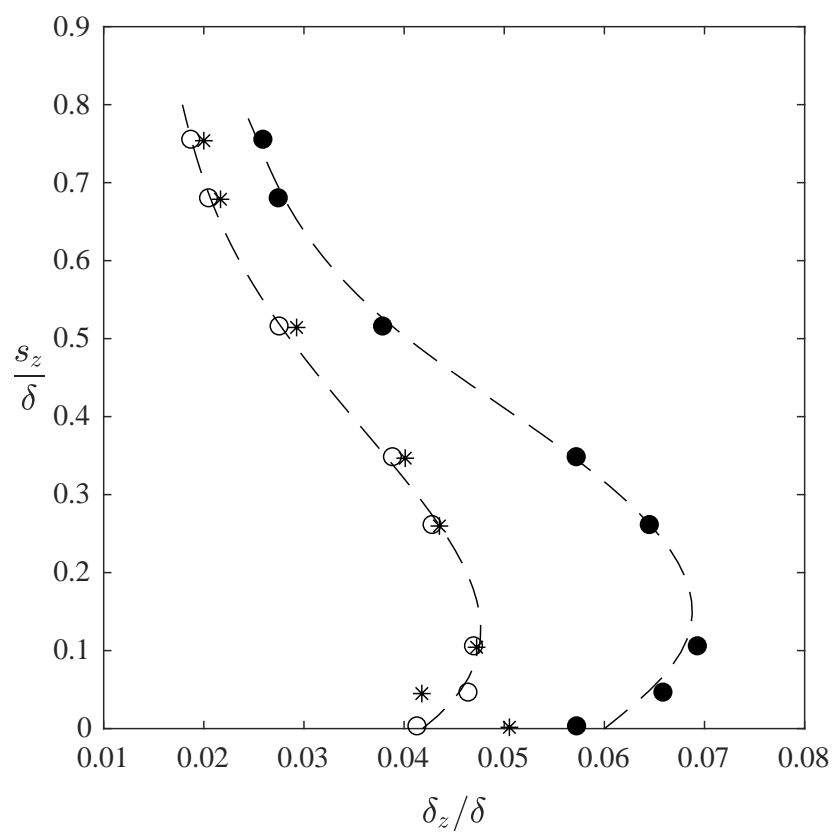

Figure 8. Vertical plume half-width $\delta_{z}$ (normalised by $\delta$ ) obtained by interpolating the distributions of mean $(O)$ and r.m.s $(\bullet)$ of concentration. Values of $\delta_{z}$ obtained from the reflected Gaussian model fit are shown using $*$ symbols.

$350 / \delta^{+}$, the plume half-width is computed as the vertical distance between the source height and the wall-normal location (above the source height) of half the maximum concentration.

Results for $\delta_{z}$ calculated by interpolation are shown using (०) symbols in figure 8 , whereas the ones obtained by fitting the reflected Gaussian model are shown in $(*)$ symbols in figure 8 for comparison. Both methods give similar results for $s_{z} / \delta \geq 0.1$ but there are clear differences when the source is close to the surface. Since the reflected Gaussian model did not yield accurate results for ground level sources, here we use the interpolation method to determine $\delta_{z}$ for all source heights.

Similarly, one can also determine $\delta_{z}$ from the r.m.s. profiles and are plotted in figure 8. The half-width from a r.m.s. profile is higher than that from a mean profile, as seen in figure 8 . To our knowledge, this is the first time that such distinction in the plume-widths is reported. As discussed before in figure 7 it is expected that $\delta_{z}$ decreases with source height and a similar trend is seen in $\delta_{z}$ values as obtained from the r.m.s profiles. Note that, $\delta_{z}$ used in the rest of the paper refers to the one computed from the mean concentration data, if not specified otherwise.

Once $\delta_{z}$ values are obtained, it is possible to examine how well the reflected Gaussian model fitted the mean concentration statistics for different $s_{z}$. For this, a local coordinate system is defined about $s_{z}$ as $\xi=z-s_{z}$ and all statistics in figures $7(\mathrm{a} \& \mathrm{~b})$ are replotted in figure 9 in the normalised form as a function of $\xi / \delta_{z}$. The respective plume half-widths for mean and r.m.s profiles are used for normalizing $\xi$, while the concentration magnitude is normalised by $C_{\max }$ or $\sqrt{\overline{c^{2}}}{ }_{\max }$, the maxima in the mean or r.m.s. at a particular $s_{z}$, respectively. It is observed that all the 

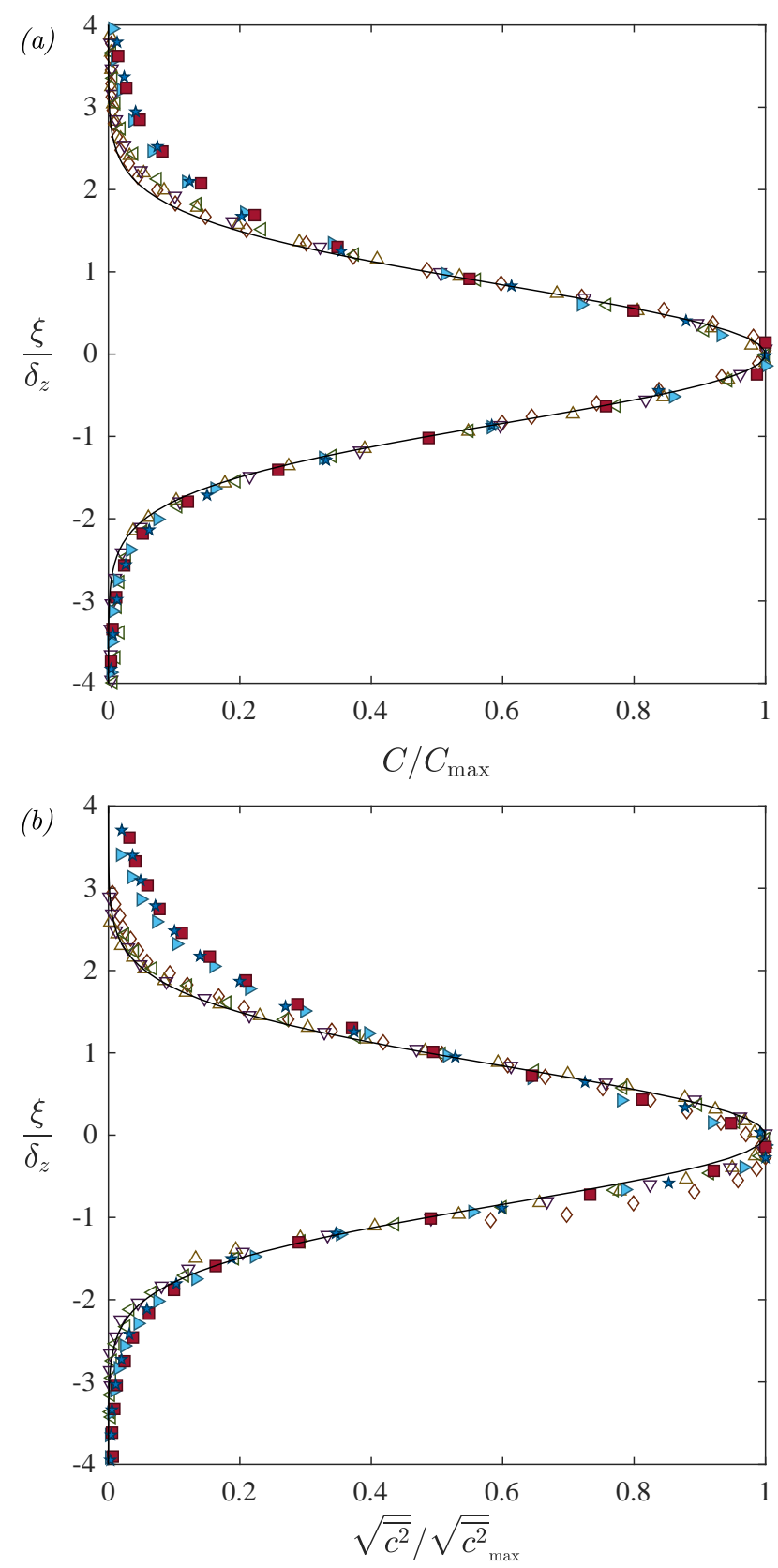

Figure 9. Normalised (a) mean and (b) r.m.s of concentration as a function of $\xi / \delta_{z}$ for all source heights. Solid line is the reflected Gaussian model. Filled symbols represent data where $s_{z}$ is in the intermittent region of the turbulent boundary layer. See table 2 for other symbols. 
normalised mean and r.m.s. profiles for $s_{z} / \delta \leq 0.33$ agree well with the reflected Gaussian model (see equation 1 ) while the remaining profiles show a deviation from the model. A closer look at these results reveal that such deviation from the reflected Gaussian model is in the outer region, where large-scale external intermittency in the turbulent boundary layer exists [Chauhan et al., 2014, and references therein]. Thus further investigation is required to explore the role of external intermittency on the plume spread, but is beyond the scope of this paper.

The results presented in figures 8 and 9 are significant as they lead to the development of a two-parameter model for the mean statistics of concentration for a point source in a turbulent boundary layer. The reflected Gaussian model as given in 1 has three parameters, namely, the coefficient $A, s_{z}$ and $\delta_{z}$, while $B$ is considered equal to $\ln (2)$. As observed in figure 8 above, one can possibly formulate an empirical relation for $\delta_{z}$ as a function of $s_{z}$. Further, it is easily seen that $A$ is a function of both $s_{z}$ and $C_{\max }$ by substituting $z=s_{z}$ in eqn. 1 because,

$$
C_{\max }=A\left[\exp \left\{-\ln (2)\left(4 s_{z}^{2} / \delta_{z}^{2}\right)\right\}\right] \text {. }
$$

This implies that the three parameters in the reflected Gaussian model can be reduced to two parameter formulation using the maximum mean concentration $\left(C_{\max }\right)$ and the source height $\left(s_{z}\right)$. The same applies for r.m.s. of concentration. Thus it is possible to predict the statistics of concentration once the source height and the mean quantities on the centreline of the plume at a given $s_{x}$ are known since the maxima, $C_{\max }$ and $\sqrt{\bar{c}^{2}}$ max are observed on the centreline of the plume. At present we refrain from formulating an expression for $\delta_{z}$ as a function of $s_{z}$ as further measurements are required to characterize the streamwise behaviour, i.e. $\delta_{z}=\delta_{z}\left(s_{z}, x, *\right)$, where $*$ represents any other variable(s). These measurements are currently being performed.

\section{Probability density functions}

Another quantity of interest in concentration measurements is the probability density and its distribution, which describes the likelihood of certain concentration level to occur at a particular point. Figure 10 shows the probability density functions (PDFs), $\mathcal{P}(\tilde{C})$ on the centreline of the plume for all source heights as a function of $\tilde{C} / \sqrt{\overline{c^{2}}}$. For the sources in the outer region, the PDF shows exponential like behaviour for $0.1 \leq s_{z} / \delta \leq 0.3$ as indicated by the linear behaviour in figure 10, where log-scale is used for the ordinate. The PDF does not show exponential behaviour when the source is positioned in the inner region $\left(s_{z} / \delta=32 / \delta^{+}\right.$and $\left.350 / \delta^{+}\right)$and the intermittent region of boundary layer, i.e., $s_{z} / \delta>0.3$.

To further explore the variations in the behaviour of PDF, a specific source height, $s_{z} / \delta=0.1$ is chosen and PDFs at several wall-normal locations are plotted in figures 11 (a) and 11 (b) as functions of $\tilde{C}$ and $\tilde{C} / \sqrt{\overline{c^{2}}}$, respectively. Blue dashed lines represent PDFs at locations above the source height, $z \leq s_{z}$ while red lines represent those below the source height. It is observed that at most locations, the PDFs exhibit exponentiallike behaviour for high concentration magnitude, i.e. $\mathcal{P}(\tilde{C}) \sim \exp (-b \tilde{C})$, where $b$ is the decay exponent; however with different slopes. This is better seen in figure 11(b) where the PDFs with identical decay exponents appear to collapse onto a single line when plotted as a function of $\tilde{C} / \sqrt{\overline{c^{2}}}$. The magnitude of the slope indicates the decay exponent of PDF at a known $z$-location. Here, $b$ is estimated from a linear 


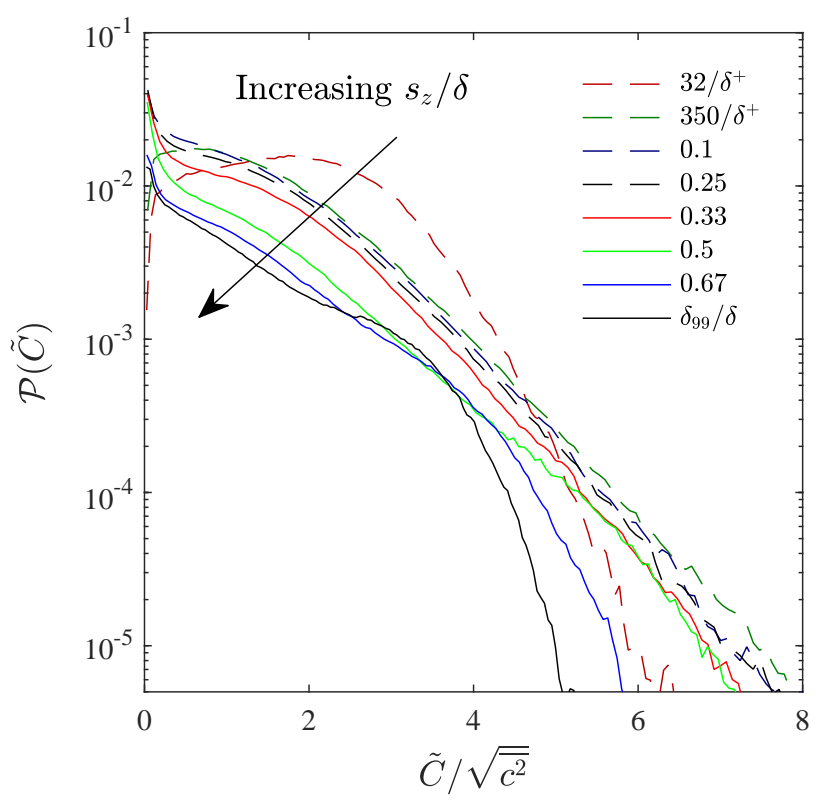

Figure 10. Probability density functions of concentration $\tilde{C}$ normalised by its r.m.s, $\sqrt{\overline{c^{2}}}$ on the centreline of the plume released at different heights in the boundary layer.

fit between $\mathcal{P}(\tilde{C})$ and $\tilde{C}$ in the range, $\tilde{C}>C_{\max }$. This procedure is repeated for all $z$-locations and the decay exponents are plotted in figure 12(a). Close to the wall, $b$ has higher magnitude corresponding to weak probability of very high concentrations. The magnitude of $b$ decreases with $z$ before seemingly becoming invariant over a finite region of the plume over an approximate region of $s_{z}-\delta_{z} \leq s_{z}+\delta_{z}$, as can be seen by comparing figures 12 (a) and (b). It is noted that this region also approximately coincides with the log-region, $0.04 \leq z / \delta \leq 0.15$. Further away in the boundary layer, the decay exponent again increases.

\section{Conclusion}

An experimental procedure is successfully implemented for simultaneous measurement of velocity and concentration at different source heights in a high Reynolds number turbulent boundary layer. It is observed that the photo-ionisation detector drifted linearly with time and hence, particular care is taken in calibrating the PID sensor to obtain reliable concentration measurements. The influence of discharge tube and PID pump on the mean statistics of turbulent boundary layer has been studied and it is found that the operation of PID has negligible influence on the flow but the discharge tube has non-negligible impact of about $\pm 5 \%$ on turbulence intensity, particularly for the sources close to the wall. The distributions of mean and r.m.s. of concentration fluctuations showed reflected Gaussian behaviour in agreement with previously reported results. However for point sources in the intermittent region of TBL there is clear deviation from the reflected Gaussian model. The vertical plume half-width $\left(\delta_{z}\right)$ is found to depend on the location of point source in the TBL and the r.m.s. profiles have wider spread (higher value of $\delta_{z}$ ) in comparison to the mean 

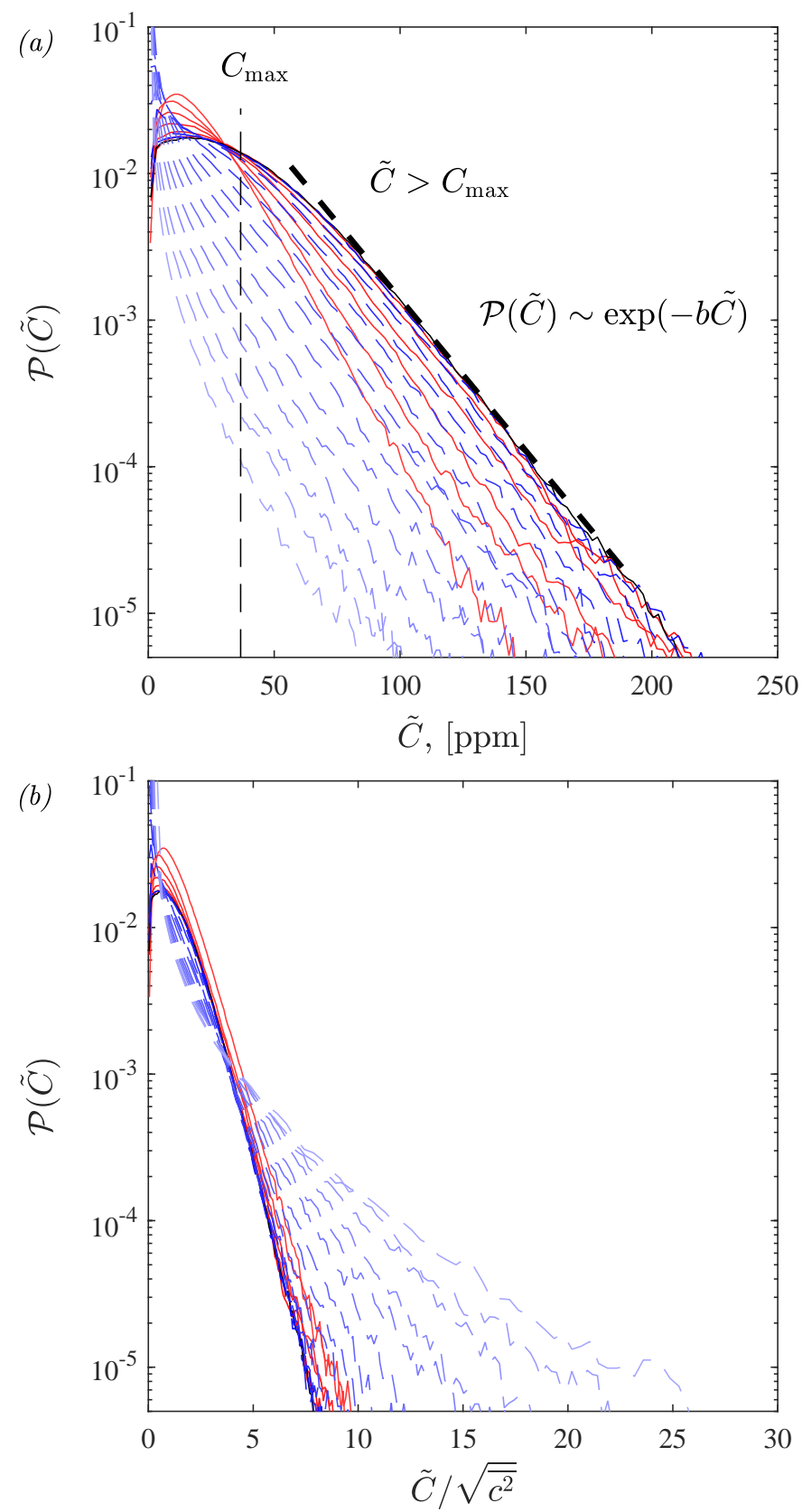

Figure 11. Probability density functions (PDFs) as a function of: (a) concentration $\tilde{C}$ at different wall-normal locations for $s_{z} / \delta=0.1$. Vertical dashed line marks the maxima in mean Concentration. The exponential behaviour of PDF is shown as the black dashed line for $\tilde{C}>C_{\max }$. The blue dashed lines show the PDFs at locations above the source height, $z \geq s_{z}$ and the red lines represent those below the source height, $z \leq s_{z}$; (b) $\tilde{C} / \sqrt{\overline{c^{2}}}$. 

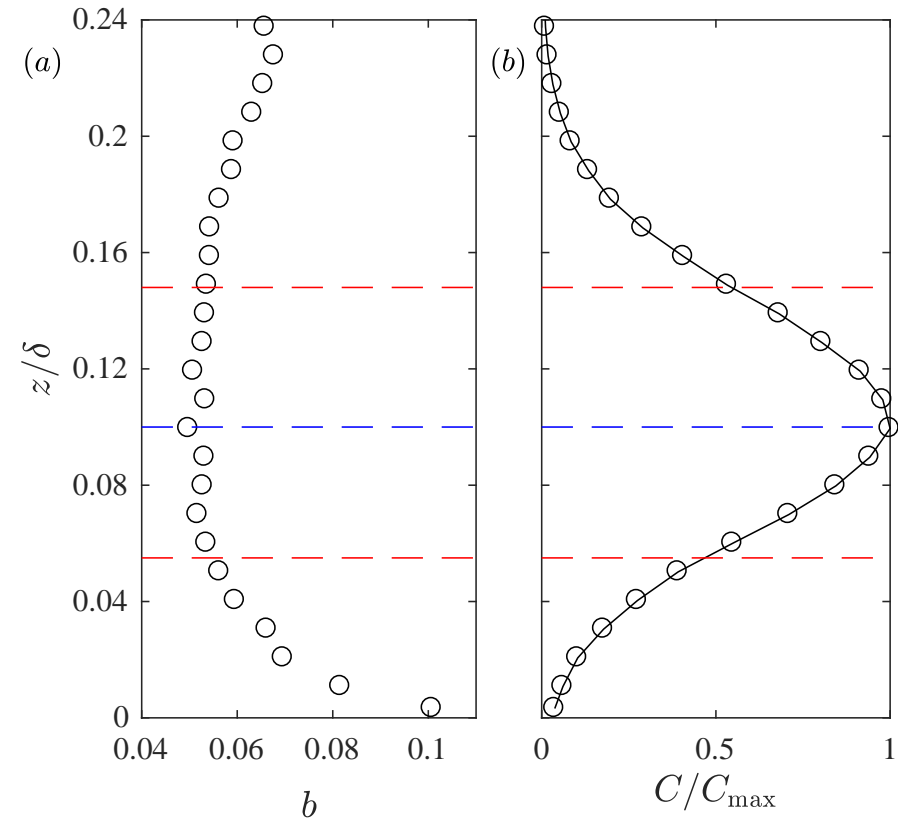

Figure 12. (a) Variation of decay exponent (b) in the exponential distributions of PDFs and (b) mean concentration, $C / C_{\max }$ as a function of normalised wallnormal distance $(z / \delta)$. The blue dashed line marks the location of the point source and the red dashed lines are drawn at $s_{z}-\delta_{z}$ and $s_{z}+\delta_{z}$

concentration profiles. The characteristics of probability density functions of high concentration is observed to depend on the wall-normal location; it has exponential behaviour in the outer-region $(0.1 \leq z / \delta \leq 0.33)$ of the TBL and a non-exponential behaviour elsewhere. Preliminary results indicate that PDFs in the log-region have identical decay exponents and further investigation is required to affirm this result.

\section{Acknowledgements}

We thank Mr. Matthew Borkowski at Aurora Scientific for his technical assistance in using the PID.

\section{References}

G. M. Chandler. Development of fast-response tracer gas sensors for use in fullscale atmospheric dispersion field trials. part I: Hardware component. part II: Field testing component. Technical report, Technical Report for Defence Research Establishment Suffield (DRES-CR-04-92), S \& J Engineering, Inc., Scarborough, Ontario, 1991.

K. A. Chauhan, H. M. Nagib, and P. A. Monkewitz. Criteria for assessing experiments in zero pressure gradient boundary layers. Fluid Dyn. Res., 41:021404, 2009.

K. A. Chauhan, J. Philip, C. M. de Silva, N. Hutchins, and I. Marusic. The turbulent/non-turbulent interface and entrainment in a boundary layer. J. Fluid Mech., 742:119-151, 2014. 
J. E. Fackrell. A flame ionisation detector for measuring fluctuating concentration. $J$. Phys. E: Sci. Instrum., 13(8):888, 1980.

J. E. Fackrell and A. G. Robins. Concentration fluctuations and fluxes in plumes from point sources in a turbulent boundary layer. J. Fluid Mech., 117(1):26, 1982.

Z. Harun, J. P. Monty, R. Mathis, and I. Marusic. Pressure gradient effects on the large-scale structure of turbulent boundary layers. J. Fluid Mech., 715:477-498, 2013.

N. Hutchins and I. Marusic. Evidence of very long meandering features in the logarithmic region of turbulent boundary layers. J. Fluid Mech., 579:1-28, 2007a.

N. Hutchins and I. Marusic. Large-scale influences in near-wall turbulence. Phil. Trans. R. Soc. A, 365:647-664, 2007b.

N. Hutchins, T. B. Nickels, I. Marusic, and M. S. Chong. Hot-wire spatial resolution issues in wall-bounded turbulence. J. Fluid Mech., 635:103-136, 2009.

P. M. Ligrani and P. Bradshaw. Spatial resolution and measurement of turbulence in the viscous sublayer using subminiature hot-wire probes. Exp. Fluids, 5(6):407-417, 1987.

I. Marusic, B. J. McKeon, P. A. Monkewitz, H. M. Nagib, A. J. Smits, and K. R. Sreenivasan. Wall-bounded turbulent flows at high Reynolds numbers: Recent advances and key issues. Phys. Fluids, 22(6):065103, 2010.

I. Marusic, K. A. Chauhan, V. Kulandaivelu, and N. Hutchins. Evolution of zeropressure-gradient boundary layers from different tripping conditions. J. Fluid Mech., 783:379-411, 2015.

M. M. Metzger and J. C. Klewicki. Development and characterization of a probe to measure scalar transport. Meas. Sci. Tech., 14(8):1437, 2003.

C. Nironi, P. Salizzoni, M. Marro, P. Mejean, N. Grosjean, and L. Soulhac. Dispersion of a passive scalar fluctuating plume in a turbulent boundary layer. Part I: Velocity and concentration measurements. Bound. Layer Meteorol., 156(3):415-446, 2015.

A. J. Smits, B. J. McKeon, and I. Marusic. High-Reynolds number wall turbulence. Annu. Rev. Fluid Mech., 43:353-375, 2011a.

A. J. Smits, J. Monty, M. Hultmark, S. C. C. Bailey, N. Hutchins, and I. Marusic. Spatial resolution correction for wall-bounded turbulence measurements. J. Fluid Mech., 676:41-53, 2011b.

K. M. Talluru, V. Kulandaivelu, N. Hutchins, and I. Marusic. A calibration technique to correct sensor drift issues in hot-wire anemometry. Meas. Sci. Tech., 25(10): 105304, 2014.

E. Yee, P. R. Kosteniuk, G. M. Chandler, C. A. Biltoft, and J. F. Bowers. Statistical characteristics of concentration fluctuations in dispersing plumes in the atmospheric surface layer. Bound. Layer Meteorol., 65(1-2):69-109, 1993.

\section{Appendix: Correcting the drift in PID during boundary layer experiments}

One of the experimental challenges we faced during the operation of PID is the drift in its output voltage. It is observed that PID voltage drifted slowly with time and we believe that it is primarily due to gradual increase in the background concentration 


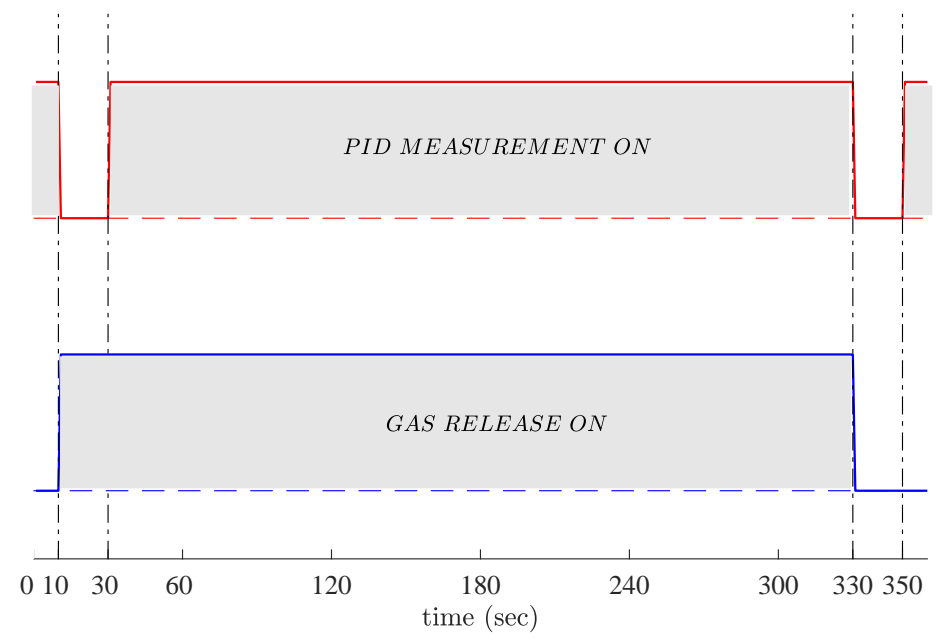

Figure 13. Timing diagram of Gas release (blue lines) and the PID measurement (red lines). The vertical lines mark the ON/OFF transitions. Grey shaded regions indicate the 'ON' state.

in the closed-loop wind tunnel used in the present study. Other factors contributing to drift could be sensitivity of internal electronics in the PID to dust, temperature and humidity. In order to accurately measure concentration not influenced by drift, an experimental procedure adopted during our measurements is described here.

A timing diagram which shows duration over which voltage output from the PID is recorded is presented schematically in figure 13. The figure also shows the relative times when the gas output from the mass flow controller is either on or off and thereby controlling the release of tracer gas from the discharge tube. At $t=$ 0 , the background concentration is measured for a duration of $10 \mathrm{sec}$ before the gas is released at $t=10 \mathrm{sec}$. A sampling duration of $10 \mathrm{sec}$ was found to be adequate to obtain converged background concentration. Once the gas is being discharged, a 20 second wait is imposed to establish steady gas flow in the tubing. The actual measurement of concentration over a 300 second period between $t=30$ and $330 \mathrm{sec}$ is recorded. Thereafter, the gas release is turned off and again after a wait period of 20 seconds (to flush out the remaining gas in the tubing), the background concentration is measured for a duration of $10 \mathrm{sec}$. Thus the background concentration is obtained before and after each measurement. A fast response time of $10 \mathrm{~ms}$ and digital control of the mass flow controller allowed precise and repeatable timing of acquisition. This procedure is implemented for every measurement point in the boundary layer. Later, the information of PID voltages in combination with the pre- and post-calibrations of PID is used to correct the PID drift in three stages as explained below.

1. Intermediate calibration curves are obtained by interpolating linearly in time between pre- and post-calibrations. Example pre- and post-calibration curves for PID in one of the experiments are shown in figure 3. This step is identical to method IV discussed in Talluru et al. [2014] for correcting hotwire drift.

2. The intermediate calibration curve is then applied to the PID voltage to convert to concentration magnitude (in ppm). 
3. Finally, the linear trend in the background concentrations measured before $(0 \leq t \leq 10 \mathrm{sec})$ and after $(350 \leq t \leq 360 \mathrm{sec})$ in every measurement is removed from the data acquired between $t=30 \mathrm{sec}$ and $t=330 \mathrm{sec}$.

The above calibration procedure is applied for every wall-normal measurement and thus the drift in PID due to change in background concentration even within a short span of 360 seconds is accounted for. Overall, consistency of results for concentration statistics discussed earlier evidence the suitability of drift correction and calibration procedure adopted here. Since this is a generic technique, it is also capable of correcting drift in PID caused due to changes in temperature (similar to method III discussed in Talluru et al. [2014]) and can be applied to measurements in open-circuit wind tunnels. 